\title{
Various Optimization Techniques Performance Testing for OFDM System Design
}

\author{
Deeksha Dimri \\ Scholar of M.Tech \\ Department of ECE \\ GRD College of Engineering, Dehradun
}

\author{
Samreen Eram \\ Assistant Professor \\ Department of ECE \\ GRD College of Engineering, Dehradun
}

\begin{abstract}
The telecommunications' industry is in the hub of an absolute detonation in wireless technologies. Once absolutely military, cellular and satellite technologies are now commercially motivated by ever more challenging consumers, who are prepared for seamless communication from their household to their car, to their workplace, or even for outside activities. With this augmented demand originates a growing essential to transmit info wirelessly, quickly, and precisely. To state this need, communications engineer have collective technologies appropriate for high rate transmission by means of forward error correction FEC techniques. The latter are chiefly important as wireless communications channels WCC are far more antagonistic as opposed to wire replacements, and the need for flexibility proves especially stimulating for reliable communications.
\end{abstract}

On the whole, Orthogonal Frequency Division Multiplexing also known as OFDM is the standard being used during the course of the world to achieve the high data tariffs necessary for data concentrated applications that must now turn out to be routine.

This thesis improves the throughput of a present OFDM system by employing adaptive modulation and OFDM coding. The new system assurances to reach a target performance bit error rate BER of 10-2 over a slow time-varying disappearing channel. The system automatically changes from lower to higher modulation arrangements on individual subcarriers, reliant on the state of the quasi-stationary channel.

\section{General Terms}

AMC (Adaptive Modulation and Coding), BER (Bit Error Rate), BLER (Block Error Rate), BPSK (Binary Phase Shift Keying), CCI (Cochannel Interference), CDF (Cumulative Distribution Function).

\section{Keywords}

OFDM, Multiplexing, performance enhancement of OFDM network.

\section{INTRODUCTION}

The necessities on the organization and equalization are governed by, among other factors, on the type of modulation. For example, discrepancy demodulation usually reduces requirement for channel evaluation and equalization at the receiver. This thesis is apprehensive with how channel uncertainties are anticipated in orthogonal frequency-division multiplexing transmission systems know as (OFDM). It addresses the evaluation of symbol time offsets, transporter frequency offsets, and channel distribution. With the aid of these estimates receiver can condense the negative things which synchronization error and channel dispersion have on system performance.
Synchronization and channel evaluation in OFDM are each connected with meticulous challenges and difficulties. For OFDM-synchronization the main confront is the high understanding to frequency offsets as compared to single transporter systems. Although timing necessities for OFDM may not be as tight as for single transporter systems, OFDM prompts for symbol synchronizers which do not bear a resemblance to traditional synchronization schemes for single transporter systems by their construction. For OFDM channel estimators the main confront is to find high presentation yet implementable estimator structures.

For an amount of wireless applications OFDM has been or is presently being investigated. Recently, European Telecommunications Standards Institute known as ETSI has based some european broadcast standards on OFDM. First, the digital audio broadcasting (DAB) set, and the worldly digital video broadcasting (DVB) set, use OFDM. Discrepancy demodulation reduces the need for a channel detector and channel equalizer in DAB receivers. Aimed at the comprehensibly demodulated DVB signals channel equalization is compulsory. Mutually audio and video receivers need to synchronize their oscillators and clocks to the transmitters. DAB signals assist synchronization by repeatedly inserted null symbols while the DVB standard defines a conduct pattern consisting of pilot tones and speckled, boosted pilot symbols.

\section{MODULATION WITH THE INVERSE FFT}

The modulation of data into a composite waveform occurs at the Inverse Fast Fourier Transform (IFFT) stage of the transmitter. Here, the modulation system can be selected entirely separately of the definite channel being used and can be selected based on the channel necessities. In fact, it is probable for each entity sub-carrier to use a unusual modulation system. The role of the IFFT is to transform each sub-channel onto the suitable carrier. The basic knowledge of OFDM is to blowout a wideband high-data-speed bit-stream over a huge number of narrow band low data speed subchannels. In conservative frequency division multiplex (FDM) systems, sub channels are entirely alienated in the frequency domain; consequently they do not obstruct with each other. For OFDM systems, there exists overlie between dissimilar sub channels and orthogonality is definite by correctly choosing transmitter and receiver filters.

\section{OFDM BASED SYSTEMS 3.1 MIMO}

A MIMO system is distinct as a system with various antennas at the receiver and transmitter ends. Traditionally, various antennas were engaged to figure the emission illustration of the antenna pattern, using a procedure known as beam 
forming. However, various antennas at the transmitter and receiver may be used to utilize array, variety, and/or multiplexing gains $[4,14]$. In latest years, techniques that broadcast over spatially uncorrelated antennas have established broad concentration from the examiner group of people due to their prospective to enlarge the dependability or the data rate of the wireless link. Spatial multiplicity and multiplexing are efficient techniques to enlarge toughness and the data rate in wireless systems requiring low difficulty. MIMO transmission schemes that presuppose channel awareness at the receiver, but not at the transmitter, mostly distribute spatial diversity or multiplexing gains [4, 14].

\subsection{Adaptation and Scheduling}

Adaptive transmission and channel-aware preparation are techniques that use channel knowledge at the transmitter end. On the basis of accessible channel sounding techniques, it is moderately ordinary to assume robust channel estimates at the receiver. Nevertheless, to have the channel approximations at the transmitter is cumbersome in non-reciprocal channels as in Frequency Division Duplex (FDD). In such arrangements, the channel is projected at the receiver and a course with the Channel State Information (CSI) is repaid to the transmitter via the feedback channel. Keep posted the CSI is liable to imperfections such as channel estimation errors, quantization errors, and feedback delays [KVC02]. More specifically, in high mobility scenarios, the rapid channel distinction causes the channel information controlled in the response to become out dated.

\subsection{Cyclic-Prefix}

The cyclic prefix is created so that each OFDM symbol is headed by a replica of the finished part of that equivalent representation. Diverse OFDM cyclic prefix lengths are existed in several systems. For example in LTE a standard length and a stretched length are available and later Release 8 a third extended length is also comprised, though not generally used. In this report emphasis is made on how the diverse parts of this thesis are interrelated. Particularly, the selection of OFDM signal models, the norms, etc. are underlined. In this section the understanding of OFDM systems to synchronization faults and the synchronization contributions of this thesis are sketched. Section 3 talks the outcome of channel dispersion on OFDM systems and summaries the channel estimation idea. Lastly, Section 4 of this preface reviews the more significant contributions of this paper.

\subsection{The Effects of Time and Frequency Offset}

In OFDM schemes orthogonality of the subcarriers is vital. While the cyclic prefix is a means to keep orthogonality in a dispersive network, symbol time and carrier frequency offsets can too cause the harm of subcarrier orthogonality. If not remunerated for, they can bound the show of an OFDM system since they cause inter-carrier interference (ICI) and inter-symbol interference (ISI). In the figure 1 beneath the symbol time offset necessities are displayed. The transmitted OFDM symbol is the acyclic prefix whose length surpasses that of the channel impulse response (top). The cyclic prefix and the symbol's tail are equal (the dark areas). As long as a receiver detentions OFDM symbol initial in the allowable region, the OFDM symbol seems cyclic, orthogonality is preserved, and ISI and ICI are dodged (bottom).

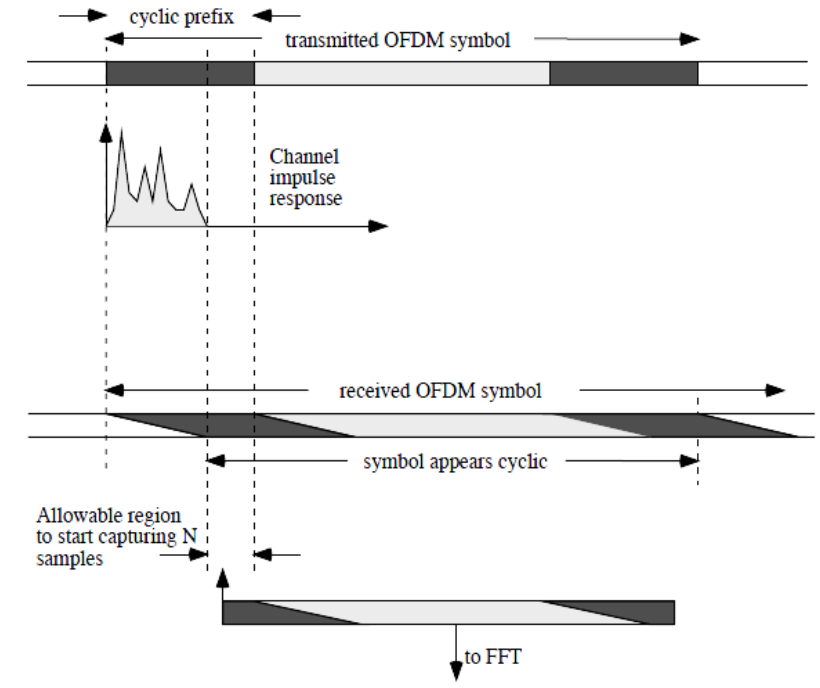

Fig 1: Symbol Time Offset

A lesser time offset is interpreted by the receiver DFT into a linear phase distortion through the subcarriers without producing ISI or ICI. The left portion of Figure 1 displays the result of a minor time offset on a transmitted signal which is only exaggerated by synchronization errors neither channel dispersal nor additive Gaussian noises alter symbols. It displays the complex-valued data codes after the receiver demodulation by the DFT.

\subsection{Symbol Synchronization}

An enormous point of attention is given to symbol synchronization in OFDM systems. Conversely by using a cyclic prefix the timing necessities are relaxed somewhat. The purpose is to know when the symbol starts. The impact of timing errors has been analyzed. A timing offset gives grow to a phase rotation of the sub carriers this phase rotation is primary on the edges of the frequency band. If a timing error is little enough to keep the channel impulse reaction within the cyclic prefix, the orthogonality is maintained. In that situation a symbol timing delay can be show as a phase shift represent by the channel and the phase rotations can be assumed by a channel estimator. ISI will arise if time shift is larger than the cyclic prefix. Synchronization technique, with modifications, is appropriate to OFDM signals transmitted by amplitude modulation. This algorithm keeping 3 phases power detection, coarse synchronization and fine synchronization.

1) Power detection detects whether or not an OFDM signal is present by measuring the usual power and compares it to a threshold.

2) Coarse synchronization is used to obtain synchronization alliance to within \pm 0.5 samples. This performance is not suitable, but this phase serves to simplify the track algorithm, which can visualize that the timing error is small. The coarse synchronization is done by correlate the recognized signal to a copy of the transmitted synchronization signal. To find the maximum point of this connection with enough accuracy, a digital filter is used to provide interpolated data rate at four times the original data rate.

3) In the line synchronization, the sub channels with pilots are stable with the expected channel obtained from pilots. As the coarse synchronization insure that the timing error is less than \pm 0.5 , the channel impulse reply 
is within the cyclic prefix. The left phase errors on the pilot sub channels are due to timing error and can be predictable by linear regression. Carrier phase noise is produced by inadequacies in the transmitter and receiver oscillators. For a frequency-selective channel, 110 difference can be complete between the phase rotation obtainable by a timing fault and a carrier phase offset [11]. The training of the effect of carrier phase noise is done in [13]. There it is presented as a Wiener process $\Theta(\mathrm{t})$ with $\mathrm{E}\{\Theta(\mathrm{t})\}=0$ and $\mathrm{E}\{(\Theta(\mathrm{tu},+\mathrm{t})-\Theta(\mathrm{tu}))\}=$ $47 \pi \beta$ t. where $\beta($ in $\mathrm{Hz})$ represents the one-sided $3 \mathrm{~dB}$ line width of the Lorentzian power density spectrum of the free-running carrier generator. The filth in SNR..i.e. tile increase in SNR desirable to recompense for the error, can be estimated by:

$D(d B) \approx \frac{11}{6 \ln 10}\left(4 \pi N \frac{\beta}{N_{u}}\right)$

Eq. 1

\subsection{Sampling Frequency Synchronization}

The conventional continuous - time signal is sampled at times resolute by the receiver clock. There are two categories of means of trade with the disparity in sampling frequency. In synchronized-sampling systems a timing algorithm reins a voltage - controlled crystal oscillator in command to align the receiver clock with the transmitter clock. The additional process is non-synchronized sampling where the sampling rate ruins stationary, which needs post-processing in the digital domain. The outcome of a clock frequency offset is double: the handy signal component is alternated and attenuated and. in addition, ICI is introduced. In [14] the biterror rate presentation of a non-synchronized sampled OFDM system has been examined. It is shown that non-synchronized sampling systems are much additional subtle to a frequency offset related with a synchronized-sampling system. For non-synchronized sampling systems, it was shown that the filth (in $\mathrm{dB}$ ) due to a frequency sampling offset depends on the square of the carrier index and on the square of the comparative frequency offset. Errors in the sampling frequency for DMT systems have been examined.

\section{RESULTS AND ANALYSIS}

Certain modification is made in wireless communication technique that uses the OFDM technology in the case of the modification on effect of QAM on BER, SNR versus BER Plot, Cyclic Prefix effect and Power clipping. Modification is made to improve the MSE and PSNR value of signal of the real time. The algorithm is presented in the section of the thesis and in this algorithm it is shown that how it can be improved by OFDM techniques.

To start our examination, for each OFDM technique, we calculate the effect of QAM on BER, SNR versus BER Plot, Cyclic Prefix effect and Power clipping. Furthermore, in order to assess the suitability of the communication means for customer of electronics products, it is calculated the PSNR or Peak Signal-to-Noise Ratio measure. The basic main of this code is to simulate the system and observe the performance of OFDM under the encouragement of various parameters for instance system noise, channel noise and channel attenuation.

Figure 2 shows the graph between bit error rate and signal to noise ratio. In this graph it is clear that as SNR increases the value of Bit Error Rate BER decreases. This graph is showing the efficiency of an OFDM communication system. Usually BER gives the measure of the error in the bit rate which is acquired by receiver at the end of the transmission.

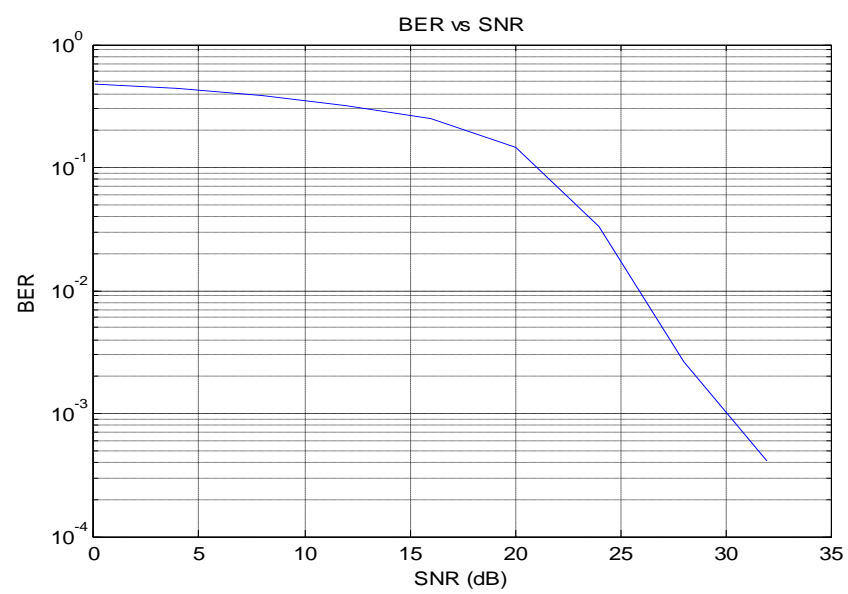

Fig 2: SNR versus BER graph for OFDM technique.

By coalescing adaptive modulation with OFDM coding, we exceeded performance expectations while ornamental the system's throughput. From figure 3 to 6 it is clearly shown how the modulation in the BPS curve happens much sooner, at an inferior SNR. This incomes that higher modulation arrangements can be enjoyed at lower SNRs finished forward error correction techniques such as OFDM coding. This enhanced throughput can be achieved without breaching the target BER.

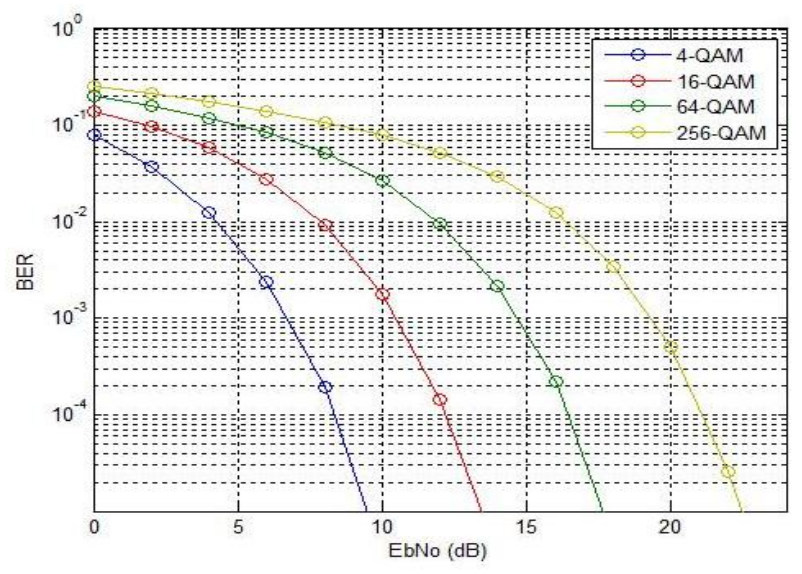

Fig 3: Effect of QAM on BER.

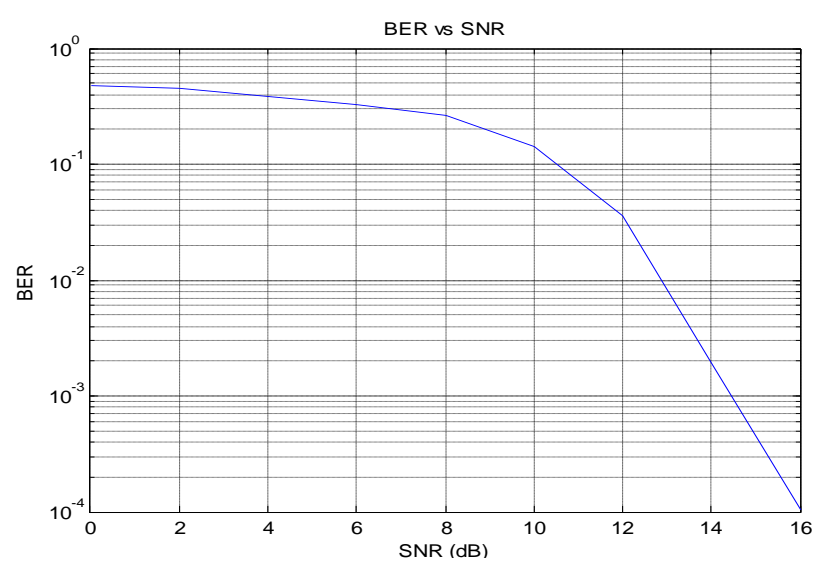

Fig 4: SNR versus BER Plot. 


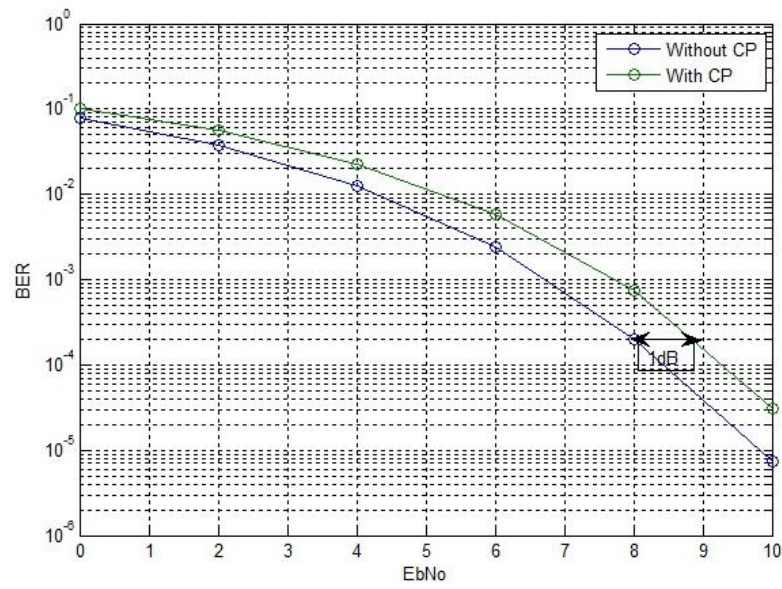

Fig 5: Cyclic Prefix effect on OFDM.

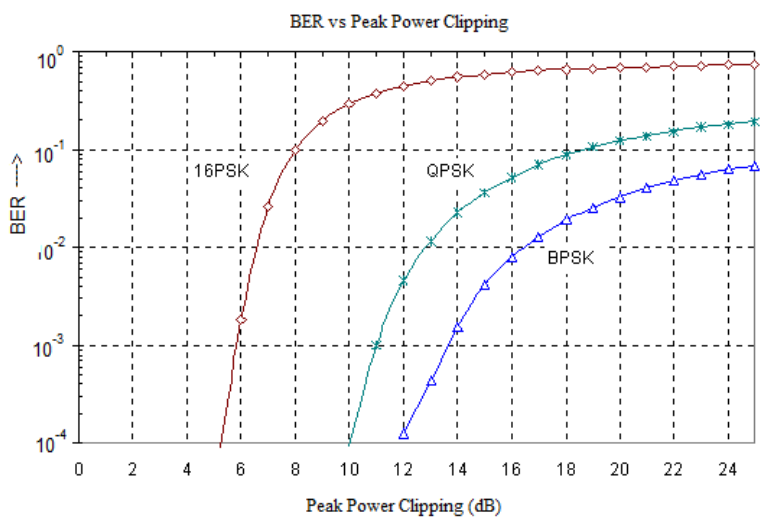

Fig 6: BER versus Power clipping graph of a OFDM system.

\section{CONCLUSION}

First we presented and simulated the wireless sluggish timevarying OFDM fading channel. We presented how its' timevarying environment (due to gesture between the transmitter and receiver) could be browbeaten to refine the system presentation and/or throughput. Once we were talented to approximation the channel, we used a justly simple targetBER adaptive inflection algorithm to achieve our goalmouth. Then, we obtained our results on the amalgamation of turbo codes and adaptive OFDM. The absence of powerful machines has not allowable to generate more bits and therefore healthier graphs. For this reason, our consequences should be deliberated preliminary.

This thesis presented that the amalgamation of turbo codes and adaptive OFDM can be influential. However, a whole coded, adaptive system would comprise a few additional wrinkles. First the system we applied can be enhanced by refining the MAP implementation from max-log-map to logmap. Such vicissitudes would only need minimal changes to the MAP cryptographer components. We have faith in that better control over the BER variations in the adaptive style can be achieved by adding a 3 bit modulation arrangement between QPSK and 16QAM. Even more controller can be attained by adding a module to vary the OFDM code's rate and puncturing designs such that assorted data rates can be conquered using the same modulation pattern (i.e. 16QAM). Not shown in our work is the nonappearance of utility of OFDM-codes when the target BER is inferior to the "error floor" of the code.
Next In the future, it would be extremely beneficial to instrument a convolutional or trellis encoder which could be used when the OFDM code use is no longer the improved unconventional. Spectrally, we could use the energy saved from transporters in the no-transmission zone to boost presentation of carriers near a swapping threshold for example. Also, enlightening the channel guesstimate practice by integrating it with the OFDM decoding process could produce some greater gains. In conclusion, to support greater operator speeds, one could appliance a channel predictor.

\section{REFERENCES}

[1] John.G. Proakis, Digital communications, 5th edition, McGraw-Hill, New York, 2000.

[2] J.-J. van de Beek et al "ML estimation of timing and frequency offset in multicarrier systems" Research Report TIJLEA Luleå. University of Technology, 1996.

[3] Timothy M. Schmidl et al "Robust Frequency and Timing Synchronization for OFDM" IEEE Transactions on Communications, No. 12, December 1997.

[4] Ye (Geoffrey) Li et al "Robust Channel Estimation for OFDM Systems with Rapid Dispersive Fading Channels" IEEE Transactions on Communications, Vol. 46, No. 7, July 1998.

[5] Michael Speth et al "Optimum Receiver Design for Wireless Broad-Band Systems Using OFDM" IEEE Transactions on Communications, Vol. 47 November 1999.

[6] Chan Kyu Kim et al "Performance of Wireless MCCDMA Antenna Array in a Fading Channel" IEEE Transaction on Communications, No.8, August 2000.

[7] Seong Taek Chung et al "Degrees of Freedom in Modulation unified View" IEEE Transactions Communications, September 2001.

[8] Severine Catreux et al "Adaptive Modulation for Broadband Wireless Networks" IEEE Communication Magazine, June 2002.

[9] Song Ni et al "On the Networking Performance of UTRA TDD and FDD CDMA Systems Using Modulation and Adaptive Beam-forming" IEEE Conference August 2003.

[10] Shengli Zhou et al "Adaptive Modulation for Multiantenna with Channel Mean Feedback" IEEE Transaction no Communications, No.5, September 2004.

[11] Pengfei Xia et al "Multi-antenna Adaptive Modulation with Beam-forming Based on Bandwidth Constrained Feedback" IEEE Transaction on Communications Vol. 53, No.3, March 2005.

[12] Hüseyin Arslan et al "Cognitive UWB-OFDM: Pushing Ultra-wideband Beyond Its Limit via Opportunistic Spectrum Usage" Journal of Communications and Networks, Vol. 8, No. 2, June 2006.

[13] Yu Zhang et al "MIMO-OFDM Systems in the Presence of Phase Noise and Doubly Selective Fading" IEEE Transactions on Vehicular Technology, Vol. 56, No. 4, July 2007.

[14] Lei Lei et al "Flow-level Performance of Opportunistic OFDM-TDMA and OFDMA Networks" IEEE 
Transactions on Wireless Communications, Vol. 7, No. 12, December 2008.

[15] Hany Elgala et al "Indoor Broadcasting via White LEDs and OFDM" IEEE Transactions on Communications, Vol. 8, No. 19, June 2009.

[16] Alan Barbieri et al "OFDM versus Single-Carrier Transmission for 100 Gbps Optical Communication" Journal of Light-wave Technology, Vol. 28, No. 17, September 1, 2010.

[17] A. Omri et al "New Transmission Scheme For MIMOOFDM System" International Journal of NextGeneration Networks (IJNGN) Vol.3, No.1, March 2011.
[18] Ludwig, Frank et al "FPGA Implementation of ZF-THP for MU-MISO-OFDM Systems" OFDM 2012, 17th International OFDM Workshop 2012.

[19] Murtuza Jeeranwala et al "Implementation of OFDM based Transreciever for IEEE 802.11a on FPGA" IJARCSSE 2014

[20] Hema Thota et al "Performance analysis of OFDM for 4G wireless systems under various fading channels" IJIREEICE 2015. 